\title{
Improving the accuracy of the muon mass and magnetic moment anomaly via the bound-muon $g$ factor
}

\author{
B. Sikora, ${ }^{1, *}$ H. Cakir, ${ }^{1}$ N. Michel,${ }^{1}$ V. Debierre, ${ }^{1}$ N. S. Oreshkina, ${ }^{1}$ N. A. Belov, ${ }^{1}$ \\ V. A. Yerokhin, ${ }^{1,2}$ C. H. Keitel, ${ }^{1}$ and Z. Harman ${ }^{1, \dagger}$ \\ ${ }^{1}$ Max Planck Institute for Nuclear Physics, Saupfercheckweg 1, 69117 Heidelberg, Germany \\ ${ }^{2}$ Center for Advanced Studies, Peter the Great St. Petersburg Polytechnic University, \\ 195251 St. Petersburg, Russia
}

(Received 29 December 2017; published 20 June 2018)

\begin{abstract}
A theoretical description of the $g$ factor of a muon bound in a nuclear potential is presented. Oneloop self-energy and multiloop vacuum polarization corrections are calculated, taking into account the interaction with the binding potential exactly. Nuclear effects on the bound-muon $g$ factor are also evaluated. We put forward the measurement of the bound-muon $g$ factor via the continuous Stern-Gerlach effect as an independent means to determine the muon's magnetic moment anomaly and mass. The scheme presented enables the increase of the accuracy of the mass by more than an order of magnitude.
\end{abstract}

DOI: 10.1103/PhysRevD.97.111301

\section{INTRODUCTION}

The physics of muons features puzzling discrepancies. The disagreement between the experimental and theoretical $g$ factor of the free muon by $3 \sigma$ represents the largest deviation from the standard model observed in the electroweak sector [1-4]. Recently, high-precision spectroscopy experiments with the muonic $\mathrm{H}$ atom yielded a value for the proton radius which strongly disagrees with that obtained from measurements on regular $\mathrm{H} \mathrm{[5,6]} \mathrm{(see} \mathrm{also} \mathrm{[7]).}$ Experiments aiming at an improved determination of muon properties can help clarify both issues and provide insight into speculative new physics.

Fast progress in the theoretical understanding and experimental precision of the bound-electron $g$ factor (see e.g., [8-16] and references therein) has enabled the most accurate determination of the mass of the electron in Penning trap $g$-factor experiments by means of the continuous SternGerlach effect $[8,17-20]$. In this paper we put forward a similar method for the extraction of the mass of the mиол by employing light muonic ions, by which we mean here bound systems solely consisting of a nucleus and a muon without further surrounding electrons. While there have been theoretical [21,22] and experimental [23-25] studies on the bound-muon $g$ factor in muonic atoms, the absence of shell electrons and the choice of light nuclei in our case

\footnotetext{
*bsikora@mpi-hd.mpg.de

harman@mpi-hd.mpg.de
}

Published by the American Physical Society under the terms of the Creative Commons Attribution 4.0 International license. Further distribution of this work must maintain attribution to the author(s) and the published article's title, journal citation, and DOI. Funded by SCOAP ${ }^{3}$. enable more accurate theoretical predictions. Also, for the sake of simplicity both on the experimental and the theoretical side, the nucleus is assumed to be spinless. Since currently the mass of the muon is only known from muonium spectroscopy $[26,27]$ and to a fractional standard uncertainty of $2.2 \times 10^{-8}$ [28,29], alternative methods for its determination are especially desirable.

When a muonic ion is subjected to a magnetic field of strength $B$, the Larmor frequency between the Zeeman sublevels depends on the magnetic moment $\mu$ of the muon by the formula

$$
\omega_{\mathrm{L}}=\frac{2 \mu}{\hbar} B=\frac{g}{2} \frac{e}{m_{\mu}} B
$$

with $e$ being the (positive) unit charge, and $g$ and $m_{\mu}$ the bound-muon $g$ factor and mass, respectively. Determining the magnetic field at the location of the ion becomes possible through a measurement of the cyclotron frequency of the ion,

$$
\omega_{\mathrm{c}}=\frac{Q}{M} B
$$

where $Q$ and $M$ are the charge and mass of the muonic ion, respectively. Thus, $m_{\mu}$ can be expressed by $M$ as

$$
m_{\mu}=\frac{g}{2} \frac{e}{Q} \frac{\omega_{\mathrm{c}}}{\omega_{\mathrm{L}}} M
$$

where the theoretical value $g_{\text {theo }}$ for the bound-muon $g$ factor is to be substituted. The quantity to be measured is the ratio of the two frequencies, $\Gamma=\omega_{\mathrm{L}} / \omega_{\mathrm{c}}$. For determining $m_{\mu}$ with a given fractional uncertainty, all the quantities 
$g_{\text {theo }}, \Gamma$ and $M$ have to be known at the same level of accuracy. For example, for the muonic ${ }^{4} \mathrm{He}^{+}$ion, the total mass $M$ is given as follows: $M=m_{\alpha}+m_{\mu}-E_{B} / c^{2}$, with $m_{\alpha}$ being the mass of the $\alpha$ particle, known to an exceptionally high relative accuracy of $1.6 \times 10^{-11}$ in atomic mass units [28], $c$ is the speed of light, and $E_{B}$ the known binding energy of the muon. Since $M$ is dominated by $m_{\alpha}\left(m_{\alpha} \approx 36 m_{\mu}\right), M$ is known to a sub-ppb accuracy. Alternatively, Eqs. (1) and (2) can be combined to yield an experimental bound-muon $g$ factor

$$
g=2 \frac{m_{\mu}}{M} \frac{Q}{e} \Gamma
$$

Such a determination of $g=2+2 a_{\mu}+\Delta g_{\text {bind }}$ constitutes an alternative access to the free muon's magnetic anomaly $a_{\mu}$ at a level at which $m_{\mu}$ is known from an independent experiment, and provided the binding contribution $\Delta g_{\text {bind }}$ can be calculated to sufficient accuracy.

In the following, we present the theory of the $g$ factor of muonic ${ }^{4} \mathrm{He}^{+}$and show that a 9-digit fractional accuracy is achievable, which corresponds to the same accuracy in the extracted muon mass or magnetic anomaly, provided the ratio of the Larmor and cyclotron frequencies can be measured with matching precision.

\section{THEORETICAL APPROACH}

The dominant Dirac value of the bound-muon $g$ factor, $g_{\mathrm{D}}$, corresponds to the tree-level Feynman diagram with the assumption of a point-like nucleus [30]. For a Dirac particle in the $1 s$ state of an ion with a charge number $Z$ it is $g_{\mathrm{D}}=\frac{2}{3}+\frac{4}{3} \sqrt{1-(Z \alpha)^{2}}$, where $\alpha$ is the fine-structure constant. Its numerical value is given in the first row of Table I. Various effects shift the bound-muon $g$ factor: First, due to the finite size of the nucleus, the interaction potential between the muon and the nucleus deviates from a pure Coulomb potential on the $\mathrm{fm}$ scale. Therefore, the wave function of the bound muon and hence its $g$ factor is corrected. This finite size (FS) correction to the bound-muon $g$ factor can be expressed with the nuclear root-mean-square radius $r_{\mathrm{RMS}}$ by the approximate formula [31] $\Delta g_{\mathrm{FS}}=\frac{8 m_{\mu}^{2}}{3}(\mathrm{Z} \alpha)^{4} r_{\mathrm{RMS}}^{2}$, in numerical agreement with [32]. As one can see on this formula, the FS correction for bound muons is more than 4 orders of magnitude larger than for bound electrons. The accuracy of $\Delta g_{\mathrm{FS}}$ is mostly

TABLE I. Various contributions to the $g$ factor of $\mu^{4} \mathrm{He}^{+}$. The abbreviations are: "eVP" " $\mu$ VP": VP due to virtual $e^{-} e^{+} / \mu^{-} \mu^{+}$pairs. The estimated uncertainty of the nuclear size effect stems from the error bar of the rootmean-square nuclear radius and the uncertainty of the nuclear charge distribution model. The uncertainty is negligible, i.e., much less than 1 in the last digit, in the case of the terms for which none is indicated. In the last row, the uncertainties due to the calculated and uncalculated (two-loop LBL) terms are given separately.

\begin{tabular}{|c|c|c|c|}
\hline Effect & Term & Numerical value & References \\
\hline Dirac value & & 1.9998579888 & {$[28,30]$} \\
\hline Finite nuclear size & & $0.0000000946(4)$ & [36] \\
\hline Nuclear polarization & & $0.0000000000(10)$ & \\
\hline \multirow[t]{2}{*}{ One-loop SE } & $(Z \alpha)^{0}$ & 0.0023228195 & \multirow[t]{2}{*}[28,37]{} \\
\hline & all-order binding & $0.0000000849(10)$ & \\
\hline \multirow[t]{4}{*}{ One-loop VP } & $e \mathrm{VP}$, Uehling & -0.0000004796 & \multirow[t]{2}{*}[38]{} \\
\hline & $e \mathrm{VP}$, magnetic loop & $0.0000001272(4)$ & \\
\hline & $\mu \mathrm{VP}$, Uehling & -0.0000000001 & \multirow[t]{2}{*}[38]{} \\
\hline & hadronic VP, Uehling & $-0.0000000001(1)$ & \\
\hline \multirow[t]{7}{*}{ Two-loop QED } & $(Z \alpha)^{0}$ & 0.0000082644 & \multirow{7}{*}{$\begin{array}{l}{[39,40]} \\
{[16,41-43]} \\
{[39-42]}\end{array}$} \\
\hline & SE-SE, $(Z \alpha)^{2}-(Z \alpha)^{5}$ & -0.0000000001 & \\
\hline & $\mathrm{S}(\mathrm{eVP}) \mathrm{E},(Z \alpha)^{2}$ & 0.0000000004 & \\
\hline & Second-order Uehling & $-0.0000000011(4)$ & \\
\hline & Källén-Sabry & -0.0000000035 & \\
\hline & magnetic loop + Uehling & 0.0000000003 & \\
\hline & uncalculated LBL & $0.0000000000(50)$ & \\
\hline$\geq$ Three-loop QED & $(Z \alpha)^{0}$ & 0.0000006106 & {$[28,44-46]$} \\
\hline \multirow[t]{3}{*}{ Nuclear recoil } & $\left(\frac{m_{\mu}}{M}\right)^{1}$, all orders in $Z \alpha$ & 0.0000060382 & {$[47]$} \\
\hline & $\left(\frac{m_{\mu}}{M}\right)^{2+},(Z \alpha)^{2}$ & -0.0000004887 & [48] \\
\hline & radiative recoil & -0.0000000047 & [49] \\
\hline Weak interaction & $(Z \alpha)^{0}$ & 0.0000000031 & {$[28,50]$} \\
\hline Hadronic contributions & $(Z \alpha)^{0}$ & $0.0000001393(12)$ & {$[28,51-53]$} \\
\hline Sum of terms calculated & & $2.0021951934(20)_{\text {calc }}(50)_{\text {uncalc }}$ & \\
\hline
\end{tabular}


limited by the uncertainty of $r_{\text {RMS }}$. The correction due to the deformation of the nuclear charge distribution was estimated using the method described in [32] and nuclear data from [33], and was found to be negligible. For the nuclear polarization correction [34,35] we assume the uncertainty to be twice the FS uncertainty.

The leading quantum electrodynamic (QED) corrections correspond to the one-loop Feynman diagrams shown on Fig. 1. These diagrams represent the electric and magnetic loop vacuum polarization (VP) corrections [Fig. 1(a) and (b), respectively] and the self-energy (SE) wave function and vertex corrections [Fig. 1(c) and (d), respectively]. We apply the method outlined in Ref. [54] for obtaining expressions for the individual terms.

The fermion loop in the VP electric loop diagram modifies the nuclear potential at distances on the scale of the Compton wavelength of the loop particle. In the free-loop approximation, this leads to the radial Uehling potential $V_{\text {Ueh }}(r)$ [55]. The effect of the Uehling term was evaluated in different ways. First, the $g$ factor contribution of the first-order Uehling diagram can be calculated as

$$
\Delta g_{\mathrm{Ueh}}=-\frac{8 m_{\mu}}{3}\left\langle a\left|V_{\mathrm{Ueh}}\right| \delta a\right\rangle
$$

where $|a\rangle$ is the bound-muon Dirac wave function and $|\delta a\rangle$ is the wave function linearly perturbed by the magnetic interaction. For a pointlike nucleus, $|\delta a\rangle$ is known analytically [56]. Since the Uehling potential does not depend on the mass of the bound particle, but only on the mass of the particle in the loop, the Uehling term can also be computed as (a)

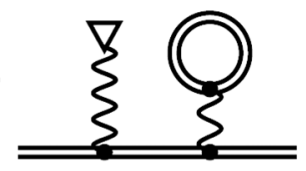

(c)

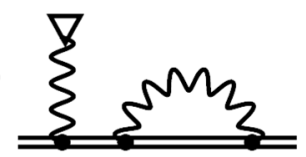

(b)

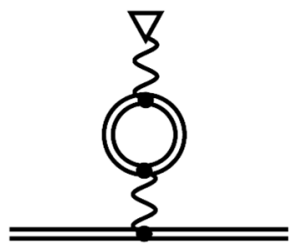

(d)

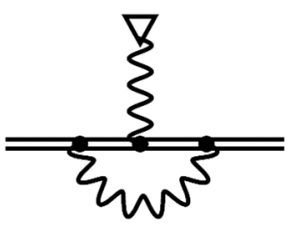

FIG. 1. Furry-picture Feynman diagrams depicting the one-loop QED corrections to the bound-muon $g$ factor: (a) the electric and (b) magnetic loop vacuum polarization corrections, and (c) the selfenergy wave function and (d) self-energy vertex correction terms. A double external line represents a muonic Coulomb-Dirac wave function, and the wave line terminated by a triangle stands for the interaction with the external magnetic field. An internal wave line represents a photon propagator, and an internal double line depicts a Coulomb-Dirac muon propagator; in vacuum polarization loops, it may also represent an electron-positron propagator.

$$
\Delta g_{\mathrm{Ueh}}=-\frac{4}{3 m_{\mu}}\left\langle a\left|\frac{\partial V_{\mathrm{Ueh}}}{\partial r}\right| a\right\rangle,
$$

according to the method described in [31,57]. In the pointlike nuclear model, the results were also compared to the exact analytical formula [38]. Values with electrons (muons) as loop particles are given as "eVP $(\mu \mathrm{VP})$, Uehling" in Table I. We note that $Z \alpha$ expansion results derived for electronic atoms cannot be straightforwardly applied to the case of muonic atoms, since they assume the loop particle to be identical to the bound particle. Furthermore, electronic VP effects would be largely overestimated by $Z \alpha$ expansion formulas, thus they need to be calculated to all orders in this parameter even at low $Z$.

The higher-order term of the electric loop VP diagram, the Wichmann-Kroll contribution, was calculated with the method of Ref. [58] and was found to be negligible. Hadronic Uehling VP corrections were estimated from the muonic Uehling term, following Ref. [59], as $\Delta g_{\text {had,VP }}=$ $0.671(15) \Delta g_{\mu \mathrm{VP}}$.

Second-order Uehling corrections, shown in Fig. 2(a), were evaluated by including the Uehling potential in the radial Dirac equation, and calculating the corresponding bound-muon wave function numerically in a B-spline representation [60], as described in Ref. [61]. Finally, the Källén-Sabry two-loop VP correction [62], illustrated in Fig. 2(b), was evaluated employing B-splines, with the effective potential given in Refs. [63,64].

The lowest-order term in the expansion of the magnetic loop VP diagram [Fig. 1(b)] corresponds to the diagram with the Coulomb-Dirac propagator replaced by the free Dirac propagator, and it does not contribute to the $g$ factor. Higher-order contributions to this diagram, such as the virtual light-by-light (LBL) scattering term, are finite. We evaluate the LBL scattering term as it was performed in Refs. $[65,66]$, with the difference that we include the finite nuclear size effect in the muon wave function. Also, we

(a)
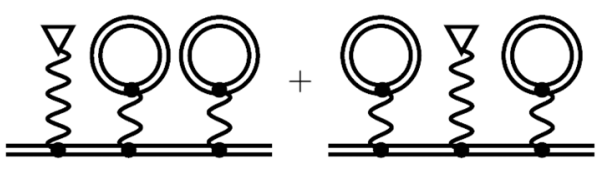

(b)

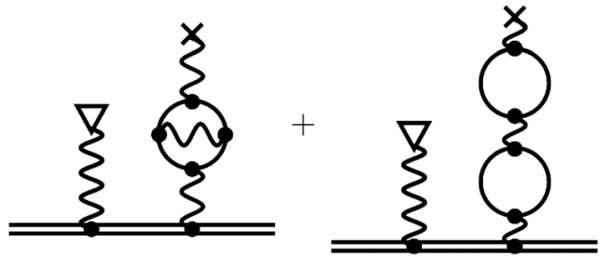

FIG. 2. Feynman diagrams of two-loop VP corrections to the bound-muon $g$ factor: (a) second-order electric-loop VP (Uehling and Wichmann-Kroll) terms, and (b) the Källén-Sabry diagrams. A single internal line represents a free Dirac propagator, and the wave line terminated by a cross stands for the interaction with the nuclear potential. 
calculate the mixed magnetic and electron loop effect by repeating the above calculation with the inclusion of the effect of the Uehling potential in the bound-muon wave function. The corresponding two-loop contribution (given in Table I as "magnetic loop + Uehling") is below the uncertainty at which we aim. We note that further two-loop corrections evaluated very recently for electronic ions, such as two-loop LBL scattering terms $[16,67]$ may also contribute significantly. Their calculation can be extended to the case of muons in a straightforward manner employing the methods of Ref. [67] or [68]. While the uncertainty due to these terms cannot be reliably given, in the table we estimate this uncertainty from the one-loop magnetic loop correction $\Delta g_{\mathrm{ML}}$ as $10 \Delta g_{\mathrm{ML}} \frac{\Delta g_{2-\text { loop }}\left[(Z \alpha)^{0}\right]}{\Delta g_{1-\text { loop }}\left[(Z)^{0}\right]^{0}}$.

In the calculation of the $S E$ wave function correction [Fig. 1(c)], the muon propagator between the magnetic interaction and the SE loop can be expressed as a spectral sum over all eigenfunctions $|n\rangle$ of the Coulomb-Dirac Hamiltonian as

$$
\sum_{n} \frac{|n\rangle\langle n|}{E_{a}-E_{n}+\operatorname{sgn}\left(E_{n}\right) i 0},
$$

with the $E_{n}$ being the eigenenergies of the $|n\rangle$ and $E_{a}$ being the eigenenergy of the reference state $|a\rangle$. The diagram needs to be split into the irreducible $\left(E_{n} \neq E_{a}\right)$ and the reducible $\left(E_{n}=E_{a}\right)$ part. The $g$-factor correction of the irreducible part can be expressed using the SE operator $\Sigma$ as

$$
\Delta g_{\mathrm{SE}, \mathrm{wf}, \text { irred }}=-\frac{8 m_{\mu}}{3}\left\langle\delta a\left|\gamma^{0} \Sigma\left(E_{a}\right)\right| a\right\rangle .
$$

Here, $\gamma^{0}$ is the time-like Dirac matrix. The irreducible part can be separated into the zero-potential contribution (free internal muon line), the one-potential contribution (free internal muon line with one interaction with the nuclear potential) and the many-potential contribution (two and more interactions with the nuclear potential). The zeropotential contribution can be written as

$$
\Delta g_{\mathrm{SE}, \mathrm{wf}, \text { irred }}^{[0]}=-\frac{8 m_{\mu}}{3}\left\langle\delta a\left|\gamma^{0} \Sigma_{2}\right| a\right\rangle .
$$

Here, $\Sigma_{2}(p)$ is the SE function of the free muon and using dimensional regularization in $d=4-\epsilon$ dimensions, it can be expressed as [69]

$$
\Sigma_{2}(p)=\delta m-\frac{\alpha}{4 \pi} \Delta_{\epsilon}\left(\not p-m_{\mu}\right)+\Sigma_{\mathrm{R}}(p),
$$

with $\delta m=\frac{3 \alpha m_{\mu}}{4 \pi}\left(\Delta_{\epsilon}+\frac{4}{3}\right)$ and $\Delta_{\epsilon}=\frac{2}{\epsilon}-\gamma_{E}-\ln m_{\mu}^{2}+\ln 4 \pi$. The cancellation of UV divergences in the one-loop SE correction to the $g$-factor has been analyzed in detail in the literature (e.g., [17,69]). For numerical calculations, it is sufficient to take into account the finite remainders of the one-loop functions defined here and in the following, since all UV divergent quantities mutually cancel. The renormalized zero-potential contribution is defined as

$$
\Delta g_{\mathrm{SE}, \mathrm{wf}, \text { irred,ren }}^{[0]}=-\frac{8 m_{\mu}}{3}\left\langle\delta a\left|\gamma^{0} \Sigma_{\mathrm{R}}\right| a\right\rangle,
$$

while the one-potential contribution is

$$
\Delta g_{\mathrm{SE}, \mathrm{wf}, \mathrm{irred}}^{[1]}=-\frac{8 m_{\mu}}{3}\left\langle\delta a\left|\gamma^{0} \Gamma_{2}^{0} V\right| a\right\rangle,
$$

with $V$ being the interaction potential of the nucleus. $\Gamma_{2}^{\nu}\left(p^{\prime}, p\right)(\nu \in\{0,1,2,3\})$ is the vertex function for free fermions and can be separated into a divergent and a regular part as [69]

$$
\Gamma_{2}^{\nu}\left(p^{\prime}, p\right)=\frac{\alpha}{4 \pi} \Delta_{\epsilon} \gamma^{\nu}+\Gamma_{\mathrm{R}}^{\nu}\left(p^{\prime}, p\right) .
$$

For details of the renormalization procedure and for expressions of $\Sigma_{\mathrm{R}}(p)$ and $\Gamma_{\mathrm{R}}^{\nu}\left(p^{\prime}, p\right)$ see Ref. [69]. The regularized one-potential term is then defined as

$$
g_{\mathrm{SE}, \mathrm{wf}, \text { irred,ren }}^{[1]}=-\frac{8 m_{\mu}}{3}\left\langle\delta a\left|\gamma^{0} \Gamma_{\mathrm{R}}^{0} V\right| a\right\rangle .
$$

The many-potential contribution was evaluated using methods described in Refs. [69,70]. It is straightforward to generalize the calculation of the Lamb-shift diagram to the many-potential contribution of the $g$-factor SE diagram. The integration over the virtual photon frequency required in the many-potential contribution was split into a lowenergy and a high-energy part. The partial-wave expansion of the low-energy part converges rapidly and does not require any extrapolation. The high-energy term converges slower. The series was computed up to Dirac angular momentum quantum numbers $|\kappa| \approx 40$, and the remainder of the series was estimated using the Richardson extrapolation method [71].

The $g$-factor contribution of the reducible SE diagram is calculated as the energy derivative of the Lamb-shift matrix element:

$$
g_{\mathrm{red}}=\left.g_{\mathrm{D}} \frac{\partial}{\partial E}\left\langle a\left|\gamma^{0} \Sigma(E)\right| a\right\rangle\right|_{E=E_{a}} .
$$

It can be again split into the zero- and many-potential contributions. While the zero-potential contribution is UV divergent, the one-potential part is finite and can therefore be included in the many-potential term.

The SE vertex correction [Fig. 1(d)] can be expressed as $\Delta E_{\text {ver }}=-e\left\langle a\left|\gamma^{0} \boldsymbol{\Gamma} \cdot \mathbf{A}\right| a\right\rangle$, with $\boldsymbol{\Gamma}$ being the 3-vector component of the vertex function. This expression can also be split into the zero- and many-potential contributions. The renormalized zero-potential contribution can be calculated in momentum space, using the magnetic vector potential $\mathbf{A}\left(\mathbf{p}^{\prime}-\mathbf{p}\right)=-\frac{i}{2}(2 \pi)^{3} \mathbf{B} \times \nabla_{\mathbf{p}} \delta\left(\mathbf{p}^{\prime}-\mathbf{p}\right)$, and can be expressed as [13] 


$$
\begin{aligned}
\Delta g_{\mathrm{ver}}^{[0]}= & -2 i m \int \frac{\mathrm{d}^{3} p}{(2 \pi)^{3}} \int \mathrm{d}^{3} p^{\prime} \bar{a}(\mathbf{p}) \\
& \times\left(\nabla_{\mathbf{p}^{\prime}} \delta\left(\mathbf{p}-\mathbf{p}^{\prime}\right) \times \boldsymbol{\Gamma}_{R}\left(p, p^{\prime}\right)\right)_{z} a\left(\mathbf{p}^{\prime}\right) .
\end{aligned}
$$

This can be further evaluated using integration by parts. For further details see Ref. [13]. We note that our numerical results for the SE terms, given at "One-loop SE" in Table I, agree well with $Z \alpha$ expansion formulas [14,41,43].

The two-loop contribution to the free-muon $\mathrm{g}$-factor [i.e., those of order $(Z \alpha)^{0}$ ] can be found in Ref. [28]. Binding corrections to the two-loop self-energy diagrams were determined up to order $(Z \alpha)^{5}$ using formulas from Refs. [14,16,41-43]. Finally, there is a two-loop diagram with a VP loop inserted in the virtual photon line of the one-loop vertex diagram (S(eVP)E in Table I); its $(Z \alpha)^{0}$ contribution is included in the free $g$-factor, and binding corrections of order $(Z \alpha)^{2}$ were computed according to Refs. [41,42].

QED corrections with three or more loops, and weak and hadronic effects can be treated in the free-muon approximation, i.e., at order $(Z \alpha)^{0}$ (see Table I for references). Their leading binding corrections are $(Z \alpha)^{2} / 6$ times smaller $[41,42]$ and thus can be neglected here.

The calculations so far have been performed in the Furry picture [72], i.e., using a static external field to describe the nucleus. The nuclear recoil contribution is the correction to the $g$ factor due to the finiteness of the nuclear mass. Formulas derived for bound electrons [47-49] are applicable also to the case of muonic ions.

\section{CONCLUSIONS}

As Table I shows, the QED binding and recoil effects treated in this work can be calculated with a $10^{-9}$ fractional accuracy for ${ }^{4} \mathrm{He}^{+}$. We anticipate that this uncertainty can be also reached for the so far unknown two-loop LBL scattering terms with existing methods $[67,68]$. This will allow for the improvement of the muon mass, or a determination of the free muon magnetic anomaly by subtracting theoretical binding effects from the measured bound-muon $g$ factor. Such experiments are challenging due to the short lifetime of the muon and several experimental problems with the production and trapping of muonic ions need to be solved. Nevertheless, in light of recent advances in the creation and precision spectroscopy of light muonic atoms $[5,6,73]$ and Penning-trap techniques such as phase-sensitive cyclotron frequency measurements $[11,74]$, this method may serve in the future as an independent muon mass or magnetic anomaly measurement technique, and along with corresponding experimental developments, will improve the mass uncertainty by more than an order of magnitude.

\section{ACKNOWLEDGMENTS}

We acknowledge insightful conversations with Jacek Zatorski. This work is part of and supported by the German Research Foundation (DFG) Collaborative Research Centre "SFB 1225 (ISOQUANT)". V. A. Y. acknowledges support by the Ministry of Education and Science of the Russian Federation Grant No. 3.5397.2017/6.7.
[1] G. W. Bennett, B. Bousquet, H. N. Brown, G. Bunce, R. M. Carey, P. Cushman, G. T. Danby, P. T. Debevec, M. Deile, H. Deng et al. (Muon (g-2) Collaboration), Phys. Rev. Lett. 92, 161802 (2004).

[2] G. W. Bennett, B. Bousquet, H. N. Brown, G. Bunce, R. M. Carey, P. Cushman, G. T. Danby, P. T. Debevec, M. Deile, H. Deng et al. (Muon (g-2) Collaboration), Phys. Rev. D 73, 072003 (2006).

[3] F. Jegerlehner and A. Nyffeler, Phys. Rep. 477, 1 (2009).

[4] M. Lindner, M. Platscher, and F. S. Queiroz, Phys. Rep. 731, 1 (2018).

[5] R. Pohl, A. Antognini, F. Nez, F. D. Amaro, F. Biraben, J. M. R. Cardoso, D. S. Covita, A. Dax, S. Dhawan, L. M. P. Fernandes et al., Nature (London) 466, 213 (2010).

[6] A. Antognini, F. Nez, K. Schuhmann, F. D. Amar, F. Biraben, J. M. R. Cardoso, D. S. Covita, A. Dax, S. Dhawan, M. Diepold et al., Science 339, 417 (2013).

[7] A. Beyer, L. Maisenbacher, A. Matveev, R. Pohl, K. Khabarova, A. Grinin, T. Lamour, D. C. Yost, T. W. Hänsch, N. Kolachevsky et al., Science 358, 79 (2017).
[8] H. Häffner, T. Beier, N. Hermanspahn, H.-J. Kluge, W. Quint, S. Stahl, J. Verdú, and G. Werth, Phys. Rev. Lett. 85, 5308 (2000).

[9] J. Verdú, S. Djekić, S. Stahl, T. Valenzuela, M. Vogel, G. Werth, T. Beier, H.-J. Kluge, and W. Quint, Phys. Rev. Lett. 92, 093002 (2004).

[10] S. Sturm, A. Wagner, B. Schabinger, J. Zatorski, Z. Harman, W. Quint, G. Werth, C. H. Keitel, and K. Blaum, Phys. Rev. Lett. 107, 023002 (2011).

[11] S. Sturm, A. Wagner, B. Schabinger, and K. Blaum, Phys. Rev. Lett. 107, 143003 (2011).

[12] T. Beier, Phys. Rep. 339, 79 (2000).

[13] V. A. Yerokhin, P. Indelicato, and V. M. Shabaev, Phys. Rev. A 69, 052503 (2004).

[14] K. Pachucki, U. D. Jentschura, and V. A. Yerokhin, Phys. Rev. Lett. 93, 150401 (2004).

[15] K. Pachucki and M. Puchalski, Phys. Rev. A 96, 032503 (2017).

[16] A. Czarnecki, M. Dowling, J. Piclum, and R. Szafron, Phys. Rev. Lett. 120, 043203 (2018). 
[17] T. Beier, H. Häffner, N. Hermanspahn, S. G. Karshenboim, H.-J. Kluge, W. Quint, S. Stahl, J. Verdú, and G. Werth, Phys. Rev. Lett. 88, 011603 (2001).

[18] S. Sturm, F. Köhler, J. Zatorski, A. Wagner, Z. Harman, G. Werth, W. Quint, C. H. Keitel, and K. Blaum, Nature (London) 506, 467 (2014).

[19] F. Köhler, S. Sturm, A. Kracke, G. Werth, W. Quint, and K. Blaum, J. Phys. B 48, 144032 (2015).

[20] J. Zatorski, B. Sikora, S. G. Karshenboim, S. Sturm, F. Köhler-Langes, K. Blaum, C. H. Keitel, and Z. Harman, Phys. Rev. A 96, 012502 (2017).

[21] V. W. Hughes and V. L. Telegdi, Bull. Am. Phys. Soc. 3, 229 (1958).

[22] K. W. Ford, V. W. Hughes, and J. G. Wills, Phys. Rev. 129, 194 (1963).

[23] D. P. Hutchinson, J. Menes, G. Shapiro, A. M. Patlach, and S. Penman, Phys. Rev. Lett. 7, 129 (1961).

[24] D. P. Hutchinson, J. Menes, G. Shapiro, and A. M. Patlach, Phys. Rev. 131, 1362 (1963).

[25] T. N. Mamedov, K. I. Gritsay, A. V. Stoykov, D. Herlach, R. Scheuermann, and U. Zimmermann, Phys. Rev. A 75, 054501 (2007).

[26] W. Liu, M. G. Boshier, S. Dhawan, O. van Dyck, P. Egan, X. Fei, M. Grosse Perdekamp, V. W. Hughes, M. Janousch, K. Jungmann et al., Phys. Rev. Lett. 82, 711 (1999).

[27] K. Jungmann, Hyperfine Interact. 127, 189 (2000).

[28] P. J. Mohr, D. B. Newell, and B. N. Taylor, Rev. Mod. Phys. 88, 035009 (2016).

[29] P. J. Mohr, D. B. Newell, and B. N. Taylor, J. Phys. Chem. Ref. Data 45, 043102 (2016).

[30] G. Breit, Nature (London) 122, 649 (1928).

[31] S. G. Karshenboim, Phys. Lett. A 266, 380 (2000).

[32] J. Zatorski, N. S. Oreshkina, C. H. Keitel, and Z. Harman, Phys. Rev. Lett. 108, 063005 (2012).

[33] R. F. Frosch, J. S. McCarthy, R. E. Rand, and M. R. Yearian, Phys. Rev. 160, 874 (1967).

[34] A. V. Nefiodov, G. Plunien, and G. Soff, Phys. Rev. Lett. 89, 081802 (2002).

[35] A. V. Volotka and G. Plunien, Phys. Rev. Lett. 113, 023002 (2014).

[36] I. Angeli and K. Marinova, At. Data Nucl. Data Tables 99, 69 (2013).

[37] J. Schwinger, Phys. Rev. 73, 416 (1948).

[38] S. G. Karshenboim, V. G. Ivanov, and V. M. Shabaev, J. Exp. Theor. Phys. 93, 477 (2001).

[39] A. Peterman, Helv. Phys. Act 30, 407 (1957).

[40] C. M. Sommerfield, Ann. Phys. (N.Y.) 5, 26 (1958).

[41] M. I. Eides and H. Grotch, Ann. Phys. (N.Y.) 260, 191 (1997).

[42] A. Czarnecki, K. Melnikov, and A. Yelkhovsky, Phys. Rev. A 63, 012509 (2000).

[43] K. Pachucki, A. Czarnecki, U. D. Jentschura, and V. A. Yerokhin, Phys. Rev. A 72, 022108 (2005).

[44] S. Laporta and E. Remiddi, Phys. Lett. B 379, 283 (1996).

[45] T. Aoyama, M. Hayakawa, T. Kinoshita, and M. Nio, Phys. Rev. Lett. 99, 110406 (2007).
[46] T. Aoyama, M. Hayakawa, T. Kinoshita, and M. Nio, Phys. Rev. Lett. 109, 111807 (2012).

[47] V. M. Shabaev and V. A. Yerokhin, Phys. Rev. Lett. 88, 091801 (2002).

[48] K. Pachucki, Phys. Rev. A 78, 012504 (2008).

[49] H. Grotch, Phys. Rev. Lett. 24, 39 (1970).

[50] A. Czarnecki, B. Krause, and W. J. Marciano, Phys. Rev. Lett. 76, 3267 (1996).

[51] J. Prades, E. de Rafael, and A. Vainshtein, The Hadronic Light-by-Light Scattering Contribution to the Muon and Electron Anomalous Magnetic Moments (World Scientific, Singapore, 2010), Vol. 20 of Advanced Series on Directions in High Energy Physics, chap. 9, p. 303.

[52] D. Nomura and T. Teubner, Nucl. Phys. B867, 236 (2013).

[53] A. Kurz, T. Liu, P. Marquard, and M. Steinhauser, Phys. Lett. B 734, 144 (2014).

[54] V. M. Shabaev, Phys. Rep. 356, 119 (2002).

[55] E. A. Uehling, Phys. Rev. 48, 55 (1935).

[56] V. M. Shabaev, in Precision Physics of Simple Atomic Systems, edited by S. G. Karshenboim and V. B. Smirnov, Lecture Notes in Physics (Springer, Berlin, 2003), p. 97.

[57] S. G. Karshenboim, R. N. Lee, and A. I. Milstein, Phys. Rev. A 72, 042101 (2005).

[58] A. G. Fainshtein, N. L. Manakov, and A. A. Nekipelov, J. Phys. B 24, 559 (1991).

[59] J. L. Friar, J. Martorell, and D. W. L. Sprung, Phys. Rev. A 59, 4061 (1999).

[60] V. M. Shabaev, I. I. Tupitsyn, V. A. Yerokhin, G. Plunien, and G. Soff, Phys. Rev. Lett. 93, 130405 (2004).

[61] N. Michel, N. S. Oreshkina, and C. H. Keitel, Phys. Rev. A 96, 032510 (2017).

[62] G. Källén and A. Sabry, K. Dan. Vidensk. Selsk. Mat. Fys. Medd. 29, 1 (1955).

[63] L. W. Fullerton and G. A. Rinker, Phys. Rev. A 13, 1283 (1976).

[64] P. Indelicato, Phys. Rev. A 87, 022501 (2013).

[65] R. N. Lee, A. I. Milstein, I. S. Terekhov, and S. G. Karshenboim, Phys. Rev. A 71, 052501 (2005).

[66] R. N. Lee, A. I. Milstein, I. S. Terekhov, and S. G. Karshenboim, Can. J. Phys. 85, 541 (2007).

[67] A. Czarnecki and R. Szafron, Phys. Rev. A 94, 060501 (2016).

[68] S. G. Karshenboim, E. Y. Korzinin, V. G. Ivanov, and V. A. Shelyuto, JETP Lett. 92, 8 (2010).

[69] V. A. Yerokhin and V. M. Shabaev, Phys. Rev. A 60, 800 (1999).

[70] W. R. Johnson, S. A. Blundell, and J. Sapirstein, Phys. Rev. A 37, 2764 (1988).

[71] L. F. Richardson, Phil. Trans. R. Soc. A 210, 307 (1911).

[72] W. H. Furry, Phys. Rev. 81, 115 (1951).

[73] R. Pohl, F. Nez, L. M. P. Fernandes, M. A. Ahmed, F. D. Amaro, P. Amaro, F. Biraben, J. M. R. Cardoso, D. S. Covita, A. Dax et al., J. Phys. Soc. Jpn. Conf. Proc., 18, 011021 (2017).

[74] S. Sturm, M. Vogel, F. Köhler-Langes, W. Quint, K. Blaum, and G. Werth, Atoms 5, 4 (2017). 